\title{
PREVALÊNCIA DA INFECÇÃO POR ENTEROPARASITAS E SUA RELAÇÃO COM AS CONDIÇÕES SOCIOECONÔMICAS E AMBIENTAIS EM COMUNIDADES EXTRATIVISTAS DO MUNICÍPIO DE CAIRU - BAHIA
}

\section{Prevalence of infection with intestinal parasites and its relation to socioeconomic and environmental conditions in extractive communities in the municipality of Cairu-Bahia}

\author{
Gabriel Muricy Cunha ${ }^{1}$, Luiz Roberto Santos Moraes ${ }^{2}$, Artur Gomes Dias Lima ${ }^{3}$, \\ Paulo Sérgio de Morais da Silveira Mattos ${ }^{4}$, Daniel Augusto Frediani ${ }^{5}$
}

Recebido em 29 de setembro de 2013; recebido para revisão em 05 de outubro de 2013; aceito em 26 de outubro de 2013; disponível on-line em 23 de novembro de 2013.

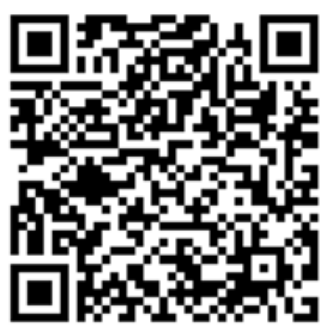

\section{PALAVRAS CHAVES:}

Parasitas Intestinais;

Saneamento Básico;

Epidemiologia.

\section{KEYWORDS:}

Intestinal Parasites;

Sanitation;

Epidemiology.

\begin{abstract}
RESUMO: As parasitoses intestinais mantêm-se como um dos maiores problemas de saúde pública em áreas rurais do Norte e Nordeste do Brasil. A pesquisal teve como objetivo realizar o inquérito da prevalência de enteroparasitas em comunidades extrativistas do município de Cairu-BA que possuíam diferenças marcantes no acesso aos serviços públicos de saneamento básico. Por meio de estudo de corte transversal com emprego do método de sedimentação espontânea foram examinados 311 indivíduos de diferentes faixas etárias. Foram levantadas informações sobre os aspectos socioeconômicos e ambientais por meio da aplicação de questionário pré-codificado padrão. Foi utilizado o software Statistical Package for Social Sciences (SPSS) para análise dos dados coletados. Na localidiade de Garapuá, 74,7\% dos indivíduos examinados estavam parasitados e 30,8\% estavam poliparasitados. As espécies enteroparasitas mais prevalentes foram: Endolimax nana (54,1\%), Giardia lamblia (29,5\%), Trichuris trichiura $(28,1 \%)$ e Entamoeba histolytica (21,2\%). Na localidade de Monte Alegre, $98,2 \%$ dos indivíduos estavam parasitados e $74,8 \%$ estavam poliparasitados. As espécies enteroparasitas mais prevalentes foram: Endolimax nana (88,3\%), Entamoeba histolytica (64,0\%), Ancylostoma sp. (55,9\%) e Entamoeba coli (51,4\%). Na localidade da Batateira, 96,3\% dos indivíduos examinados estavam parasitados e 79,6\% (43/54) estavam poliparasitados. As espécies enteroparasitas mais prevalentes foram: Endolimax nana $(87,0 \%)$, Trichuris trichiura (68,5\%), Entamoeba histolytica (66,7\%) e Ascaris lumbricoides (46,3\%).
\end{abstract}

ABSTRACT: Intestinal parasitic diseases remain one of the major public health problems in rural areas of the North and Northeast of Brazil. This research aimed to survey the prevalence of intestinal parasites in extractive communities in the city of Cairu - BA which had marked differences in access to basic sanitation public services. The survey of the prevalence of intestinal parasites and survey data on the socioeconomic and environmental aspects were performed by means of cross-sectional study. The Socioeconomic and environmental aspects was conducted through interviews at the household level, with the use of standard questionnaire semi-structured and pre-coded. The qualitative fecal examinations were performed by the technique of spontaneous sedimentation. The study population was composed of individuals of all ages residing in the village of Monte Alegre and Batateira, and children (0-14 years) living in the village of Garapuá. Examined a total of 311 individuals. The results were described with the use of the Statistical Package for Social Sciences. In the village of Garapuá, $74,7 \%$ of the individuals examined were parasitized and $30.8 \%$ were infected of three or more intestinal parasites. The species most prevalent were Endolimax nana (54,1\%), Giardia Iamblia (29,5\%), Trichuris trichiura (28,1\%) and Entamoeba histolytica (21,2\%). In the village of Monte Alegre, $98,2 \%$ of individuals were infected and $74,8 \%$ were infected of three or more intestinal parasites. The species most prevalent were Endolimax nana (88,3\%), Entamoeba histolytica (64,0\%), Ancylostoma sp. (55,9\%) and Entamoeba coli $(51,4 \%)$. In the village of Batateira, $96,3 \%$ of the individuals examined were infected and $79.6 \%(43 / 54)$ were infected of three or more intestinal parasites. The species most prevalent were Endolimax nana $(87,0 \%)$, Trichuris trichiura (68,5\%), Entamoeba histolytica $(66,7 \%)$ and Ascaris lumbricoides $(46,3 \%)$. 
* Contato com o autor:

${ }^{1}$ e-mail : gabriel_muricy@hotmail.com (G. M. Cunha )

Biomédico, Mestre em Saúde, Ambiente e Trabalho.

2e-mail : moraes@ufba.br ( L. R.S. Moraes )

PhD em Saúde Ambiental, Professor Titular em Saneamento e Participante Especial - Universidade Federal da Bahia, Programa de Pós-Graduação em Saúde, Ambiente e Trabalho - Departamento de Medicina Preventiva e Social. End. Largo do Terreiro de Jesus.

3 e-mail : parasitologista@gmail.com (A. G. Dias-Lima )

Doutor em Biologia Parasitária, Professor Titular, Universidade do Estado da Bahia.

${ }^{4}$ e-mail : parasitologiasta@gmail.com (P.S.M. S. Mattos )

Graduando em Biomedicina - Escola Bahiana de Medicina e Saúde Pública.

${ }^{5}$ e-mail : danielfrediani@hotmail.br (D. A. Frediani )

Graduando em Engenharia Sanitária e Ambiental - Universidade Federal da Bahia, Escola Politécnica.

\section{INTRODUÇÃO}

Os avanços e retrocessos nos conhecimentos em saneamento determinaram o desenvolvimento ou decadência das civilizações (MENEZES, 1984). Segundo a Organização Panamericana da Saúde (2011), o saneamento básico é a intervenção de saúde com maior impacto no desenvolvimento de uma região, na promoção da saúde individual e coletiva.

Reflexo de um contexto histórico e atual de extrema injustiça social e ambiental, as parasitoses intestinais ainda representam um grave problema de saúde pública, principalmente, em áreas pauperizadas dos países em desenvolvimento, excluídas do acesso aos serviços públicos de saneamento básico, realidade comum aos países latino americanos, africanos e asiáticos, onde persistem elevadas taxas de morbimortalidade em crianças, por doenças passíveis de prevenção (OPAS, 2011).

No Brasil, após a Constituição Federal de 1988, as medidas de saneamento básico passam a ser entendidas, constitucionalmente, como uma atividade de promoção e prevenção à saúde da população. No entanto, como legado das políticas excludentes, norteadas pela lógica do mercado, os serviços públicos de saneamento básico são ainda deficientes ou mesmo inexistentes em muitos locais (MORAES et al., 2006; TEIXEIRA, 2011).

Em 2010, menos de $50 \%$ da população rural do País possuía acesso a tecnologias adequadas de esgotamento sanitário (WHO; UNICEF, 2012). Nesse contexto, as doenças infectoparasitárias mantêm-se como um dos maiores problemas de saúde pública do País, principalmente, nas áreas rurais das regiões Norte e Nordeste.

Diretamente associadas às condições ambientais em que vivem as populações, a frequência das infecções por enteroparasitas vem sendo utilizada como indicador epidemiológico capaz de sinalizar o impacto da deficiência ou inexistência de medidas de saneamento básico sobre a saúde pública, bem como, a efetividade das intervenções sanitárias.

Este artigo é resultado de projeto de pesquisa que teve como objetivo estimar a prevalência da infecção por enteroparasitas e os fatores sociais, econômicos, culturais, sanitários e ambientais associados, em comunidades extrativistas do Município de Cairu - BA.

O projeto de pesquisa foi coordenado por equipe técnica vinculada à Universidade Federal da Bahia (UFBA), com apoio técnico e financeiro da Prefeitura Municipal de Cairu.

A escolha do foco e do contexto do artigo justifica-se devido à gravidade do problema das parasitoses intestinais, às precárias condições de saneamento básico das localidades estudadas, e à carência de ações de vigilância sanitária e ambiental.

\section{METODOLOGIA}

\section{1 ÁREA DE ESTUDO}

O município de Cairu, localizado a $308 \mathrm{~km}$ de Salvador-BA, é o único município brasileiro com espaço territorial formado por um arquipélago. 
O território municipal, com área de $460,980 \mathrm{~km}^{2}$, é composto por 26 ilhas, sendo constituído pelos distritos de Galeão, Gamboa e Velha Boipeba, e as vilas e/ou povoados: Canavieiras, Torrinhas, Tapuias, Morro de São Paulo, Zimbo, Batateira, Garapuá, Monte Alegre, Cova da Onça, além da sede do município, Cairu. As três maiores ilhas, Tinharé, Boipeba e Cairu, são os locais onde está assentada a maior parcela da população, que o censo de 2010 contabilizou em 15.374 habitantes.

Apesar da beleza cênica, a geografia do município de Cairu dificulta a universalização dos serviços públicos de saneamento básico (abastecimento de água, esgotamento sanitário, manejo de resíduos sólidos e de drenagem de águas pluviais), em especial às populações tradicionais (quilombolas, pescadores e marisqueiros) assentadas em áreas rurais de difícil acesso.

Com base nos dados do Sistema de Informação da Atenção Básica, em 2012, 82,97\% das famílias cadastradas possuíam acesso à rede pública de abastecimento de água em domicílio, $24,24 \%$ possuíam acesso à rede coletora de esgoto, e $82,28 \%$ possuíam acesso à coleta pública de resíduos sólidos (DATASUS, 2012).

No âmbito político, o município de Cairu não possui um arcabouço legal destinado a orientar a gestão dos serviços públicos de saneamento básico. Recentemente, esforços tem sido empreendidos na elaboração do Plano de Gestão Integrada de Resíduos Sólidos (PGIRS), sob coordenação da CONDER (Companhia de Desenvolvimento Urbano do Estado da Bahia), e do Plano Municipal de Saneamento Básico.

\subsection{DESENHO EPIDEMIOLÓGICO}

O estudo da prevalência de enteroparasitas e o levantamento de dados sobre variáveis socioeconômicas e ambientais foram realizados, por meio de estudo de corte transversal.

As localidades alvo do estudo (Garapuá, Batateira e Monte Alegre) foram selecionadas de forma não aleatória, por possuírem diferentes níveis de cobertura dos serviços públicos de saneamento básico, especialmente no que se refere ao abastecimento de água, esgotamento sanitário e coleta de resíduos sólidos, e semelhanças, quanto às variáveis sociais, culturais, econômicas e ambientais, o que, teoricamente, as tornam "comparáveis".

\subsubsection{População Estudada}

A população estudada foi formada por indivíduos de todas as faixas etárias residentes nas localidades quilombolas de Monte Alegre e Batateira, e crianças (0 a 14 anos) residentes na localidade de Garapuá.

Foi examinado um total de 311 indivíduos. Foram examinados 111 indivíduos da localidade de Monte Alegre, 54 indivíduos da localidade da Batateira e 146 crianças da localidade de Garapuá.

\subsection{LEVANTAMENTO DAS VARIÁVEIS SOCIOECONÔMICAS E SANITÁRIAS}

Foram realizadas entrevistas no nível domiciliar, com o emprego de questionário padrão, semiestruturado e pré-codificado.

Foram também levantadas informações sobre os aspectos demográficos (idade, sexo, endereço, tempo de residência no domicílio, no de quartos e no de moradores no domićlio), econômicos (profissão e renda mensal do chefe da família, acesso ao programa municipal cheque solidário, acesso ao programa federal bolsa família, posse de bens como fogão, geladeira, televisão e acesso a internet em domicílio), sociais (escolaridade do chefe da família, escolaridade do cuidador, escolaridade da criança, participação no programa de erradicação do trabalho infantil, tempo de amamentação da criança, uso de vermífugo e acesso a energia elétrica) e sanitários (tipologia construtiva da parede, piso e cobertura do domicílio, acesso a água encanada em domicílio, disponibilidade de pia no interior do domicílio, disponibilidade de caixa d' água no domicílio, água utilizada para consumo, água utilizada no preparo de alimentos, forma de tratamento da água utilizada para consumo, forma de armazenamento da água utilizada para consumo, existência de sanitário em domicílio, forma de acondicionamento 
do lixo domiciliar, acesso a coleta de lixo em domicílio, frequência da coleta de lixo em domicílio, destino do lixo orgânico, destino do lixo seco, local de banho e pavimento da via pública).

\subsection{COLETA DAS AMOSTRAS E ANÁLISE PARASITOLÓGICA DE FEZES}

Coletores de fezes contendo formalina a $5 \%$, previamente identificados com o nome de cada indivíduo e código alfanumérico (identificação da localidade, rua, no da casa e no de identificação do indivíduo em cada família) referente ao questionário, foram entregues em domicílio, após as orientações a respeito dos procedimentos adequados à coleta da amostra de fezes.

No dia seguinte à entrega dos coletores, foi realizada busca ativa das amostras, por meio de visitas domiciliares. As amostras entregues foram acondicionadas em caixa térmica e imediatamente enviadas ao laboratório de parasitologia da Escola Bahiana de Medicina e Saúde Pública, Campus III, localizado no bairro do Cabula, Salvador-Bahia.

\subsection{ANÁLISE PARASITOLÓGICA DE FEZES}

Foram realizados exames coproparasitológicos qualitativos, por meio da técnica de Sedimentação Espontânea, com a finalidade do diagnóstico de cistos de protozoários, ovos e larvas de helmintos.

Foi examinada apenas uma amostra de cada indivíduo estudado. Cada amostra foi analisada por três laboratoristas, de forma independente.

Visando minimizar o viés de aferição, os laboratoristas foram mantidos "cegos" com relação às condições sanitárias das localidades estudadas.

\subsection{ENTREGA DOS RESULTADOS E TRATAMENTO DOS INDIVÍDUOS PARASITADOS}

Os resultados dos exames foram entregues pelas equipes de saúde da família que atendem as comunidades, de forma individualizada, impedindo a exposição dos indivíduos estudados.

Os indivíduos parasitados foram submetidos a exame clínico e medicados com os quimioterápicos Albendazol e/ou Secnidazol.

\subsection{ANÁLISES ESTATÍSTICAS}

Para cada localidade estudada foi construído um bancos de dados em Excel (2010), contendo todas as variáveis socioeconômicas e sanitárias levantadas e os resultados laboratoriais encontrados. Os dados digitados foram devidamente conferidos (limpeza do banco).

Foi utilizado o software Statistical Package for Social Sciences - SPSS (versão 13.0) para a descrição das variáveis socioeconômicas e ambientais e dos resultados laboratoriais encontrados.

\subsection{ASPECTOS ÉTICOS}

O projeto de pesquisa foi aprovado pelo Comitê de Ética em Pesquisa da Universidade Estadual da Bahia - CEP/UNEB.

Os indivíduos interessados em participar da pesquisa assinaram o termo de consentimento livre e esclarecido.

\section{RESULTADOS}

Com relação aos aspectos socioeconômicos levantados em cada localidade que compôs a área de estudo, foi verificado que na localidade de Monte Alegre, $66,7 \%$ dos chefes de família eram analfabetos, $18,9 \%$ possuíam o ensino primário e $11,7 \%$ o ensino ginásio. Nenhum chefe de família possuía o ensino médio. Quanto à renda mensal do chefe da família, foi constatado que $45 \%$ dos chefes de família possuíam renda mensal menor do que um salário mínimo, 51,4\% possuíam renda igual ou maior que 1 salário mínimo e apenas 1 chefe de família (0,9\%) possuía renda igual ou maior a dois salários mínimos (Tabela 1 ).

$\mathrm{Na}$ localidade da Batateira, 35,2\% dos chefes de família eram analfabetos, 48,1\% possuíam o ensino primário e $16,7 \%$ possuíam o ensino ginásio. Também na localidade da Batateira nenhum chefe de família possuía ensino médio. Quanto à renda mensal do chefe da família, foi constatado que $81,5 \%$ possuíam renda menor do que um salário mínimo e $18,5 \%$ possuíam renda igual ou maior que 1 salário mínimo (Tabela 1). 
Na localidade de Garapuá, $13 \%$ dos chefes de família eram analfabetos, $37,7 \%$ possuíam o ensino primário, $28,1 \%$ possuíam o ensino ginásio, $12,3 \%$ possuíam o ensino médio e $6,8 \%$ possuíam o ensino superior. Quanto à renda, verificou-se que $52,1 \%$ dos chefes de família possuíam renda mensal menor do que um salário mínimo, 40,4\% possuíam renda igual ou maior que 1 salário mínimo e apenas $5,5 \%$ possuíam renda igual ou maior a dois salários mínimos (Tabela 1).

Com relação aos aspectos sanitários, foi verificado que na localidade de Monte Alegre, $82 \%$ dos indivíduos estudados possuíam acesso a água encanada em domicílio, 25,2\% dispunham de pia no interior do domicílio, 30,6\% residiam em moradias equipadas com caixa d'água e $48,6 \%$ residiam em domicílios equipados com vaso sanitário. $\mathrm{Na}$ população examinada, $79,3 \%$ dos indivíduos eram atendidos pelo serviço publico de coleta de lixo em domicílio (Tabela 2).

$\mathrm{Na}$ localidade da Batateira, todos os indivíduos estudados residiam em casas sem acesso a água encanada, pia no ambiente interno, caixa d'água e vaso sanitário. Entre os indivíduos estudados, $77,8 \%$ eram atendidos pelo serviço publico de coleta de lixo em domicílio de forma esporádica (Tabela 2).

Na localidade de Garapuá, 95,9\% dos indivíduos examinados possuíam acesso a água encanada em domicílio, $75,3 \%$ dispunham de pia no interior do domicílio, 53,4\% residiam em moradias equipadas com caixa d'água e 95,2\% em moradias equipadas com vaso sanitário. Todos os indivíduos examinados eram atendidos pelo serviço público de coleta de lixo em domicílio (Tabela 2).

Tabela 1: Aspectos Socioeconômicos Levantados.

\begin{tabular}{l|c|c|c}
\hline \multirow{2}{*}{ Variável } & Garapuá & Batateira & Monte Alegre \\
\cline { 2 - 4 } & $\%$ & $\%$ & $\%$ \\
\hline Escolaridade do Chefe da Família & & & $(66,7)$ \\
Analfabeto & $(13,0)$ & $(35,2)$ & $(18,9)$ \\
Ensino Primário & $(37,7)$ & $(48,1)$ & $(11,7)$ \\
Ensino Ginásio & $(28,1)$ & $(16,7)$ & $(0,0)$ \\
Ensino Médio & $(12,3)$ & $(0,0)$ & $(0,0)$ \\
Ensino Superior & $(6,8)$ & $(0,0)$ & $(45,0)$ \\
& & & $(51,4)$ \\
Renda Mensal do Chefe da Família & & $(81,5)$ & $(0,9)$ \\
$<$ que 1 salário mínimo & $(52,1)$ & $(18,5)$ & $(0,0)$ \\
Igual ou maior a 1 salário mínimo & $(40,4)$ & & \\
Igual ou maior a 2 salários mínimos & $(5,5)$ & & \\
\hline
\end{tabular}

Tabela 2: Aspectos Sanitários.

\begin{tabular}{l|l|l|l|l|l|l}
\hline Variáveis Estudadas & \multicolumn{2}{l|}{$\begin{array}{l}\text { Garapuá } \\
\text { (N=146) }\end{array}$} & \multicolumn{2}{l|}{$\begin{array}{l}\text { Batateira } \\
\text { (N=54) }\end{array}$} & $\begin{array}{l}\text { Monte } \\
\text { (N=111) }\end{array}$ \\
\hline & $\mathbf{N}$ & $\mathbf{\%}$ & $\mathbf{N}$ & $\mathbf{\%}$ & $\mathbf{n}$ & $\mathbf{\%}$ \\
\hline Água Encanada & 140 & $(95,9)$ & 0 & $(0,0)$ & 91 & $(82,0)$ \\
Pia no Domicílio & 110 & $(75,3)$ & 0 & $(0,0)$ & 28 & $(25,2)$ \\
Caixa D'água & 78 & $(53,4)$ & 0 & $(0,0)$ & 34 & $(30,6)$ \\
Vaso Sanitário em Domicílio & 139 & $(95,2)$ & 0 & $(0,0)$ & 54 & $(48,6)$ \\
Coleta de Lixo em Domicílio & 143 & $(97,9)$ & 44 & $(81,5)$ & 88 & $(79,3)$ \\
\hline
\end{tabular}


Quanto à tipologia construtiva das residências, constatou-se que na Vila de Monte Alegre, $78,4 \%$ dos indivíduos estudados residiam em casas com parede de taipa e $21,6 \%$ em casas com paredes de alvenaria com reboco. Nenhum indivíduo examinado residia em casa com parede de madeira, palha ou plástico.

Com relação ao material predominante no piso das residências, $7,2 \%$ dos indivíduos residiam em casas com piso de barro batido, $74,8 \%$ em casas com piso de cimento e $18 \%$ em casas com piso cerâmico.

Com relação ao material predominante na cobertura das residências, $35,1 \%$ dos indivíduos residiam em casas com cobertura de telha cerâmica e $64,9 \%$ residiam em casas com cobertura de telha de cimento-amianto (Eternit) (Tabela 3).

Na Vila da Batateira, $57,4 \%$ dos indivíduos estudados residiam em casas com paredes de madeira, 33,3\% residiam em casas com paredes de taipa e 9,3\% residiam em casas com paredes de palha. Nenhum indivíduo residia em casa com parede de alvenaria (Tabela 3).

Com relação ao material predominante no piso das residências, $57,4 \%$ dos indivíduos residiam em casas com piso de areia, 33,3\% em casas com piso de cimento e $9,3 \%$ em casas com piso cerâmico (Tabela 3).

Com relação ao material predominante na cobertura das residências, constatou-se que $85,2 \%$ dos indivíduos residiam em casas com cobertura de telha cerâmica e $14,8 \%$ dos indivíduos residiam em casas com cobertura de cimento-amianto (Eternit) (Tabela 3).

Na Vila de Garapuá, $74,0 \%$ das crianças examinadas residiam em casas com parede de alvenaria com reboco, 17,1\% em casas com parede de madeira e $6,8 \%$ em casas com parede de alvenaria sem reboco.

Com relação ao material predominante no piso das residências, $54,1 \%$ das crianças residiam em casas com piso cerâmico e $43,8 \%$ em casas com piso de cimento.

Com relação ao material predominante na cobertura das residências, $69,2 \%$ dos indivíduos residiam em casas com cobertura de telha cerâmica, 17,1\% em casas com cobertura de telha de cimento-amianto (Eternit), 10,9\% em casas com laje de concreto e $0,7 \%$ residia em casa com cobertura de plástico (Tabela 3).

Tabela 3. Tipologia Construtiva das Habitações nas Localidades Estudadas.

\begin{tabular}{|c|c|c|c|c|c|c|}
\hline \multirow[t]{2}{*}{ Tipologia } & \multicolumn{2}{|c|}{$\begin{array}{c}\text { Garapuá } \\
(\mathrm{N}=146)^{*}\end{array}$} & \multicolumn{2}{|c|}{$\begin{array}{c}\text { Batateira } \\
(N=54)\end{array}$} & \multicolumn{2}{|c|}{$\begin{array}{l}\text { Monte Alegre } \\
\quad(\mathrm{N}=111)\end{array}$} \\
\hline & $n$ & $\%$ & $n$ & $\%$ & $n$ & $\%$ \\
\hline \multicolumn{7}{|l|}{ Piso } \\
\hline Barro ou Areia & 0 & $(0,0)$ & 31 & $(57,4)$ & 8 & $(7,2)$ \\
\hline Cimento & 64 & $(43,8)$ & 18 & $(33,3)$ & 83 & $(74,8)$ \\
\hline Cerâmica & 79 & $(54,1)$ & 5 & $(9,3)$ & 20 & $(18,0)$ \\
\hline \multicolumn{7}{|l|}{ Parede } \\
\hline Taipa & 0 & $(0,0)$ & 18 & $(33,3)$ & 87 & $(78,4)$ \\
\hline Madeira & 25 & $(17,1)$ & 31 & $(57,4)$ & 0 & $(0,0)$ \\
\hline Palha & 0 & $(0,0)$ & 5 & $(9,3)$ & 0 & $(0,0)$ \\
\hline Bloco s/ Reboco & 10 & $(6,8)$ & 0 & $(0,0)$ & 0 & $(0,0)$ \\
\hline Bloco c/ Reboco & 108 & $(74,0)$ & 0 & $(0,0)$ & 24 & $(21,6)$ \\
\hline \multicolumn{7}{|l|}{ Cobertura } \\
\hline Laje de Concreto & 16 & $(10,9)$ & 0 & $(0,0)$ & 0 & $(0,0)$ \\
\hline Telha Cerâmica & 101 & $(69,2)$ & 46 & $(85,2)$ & 39 & $(35,1)$ \\
\hline Telha de Eternit & 25 & $(17,1)$ & 8 & $(14,8)$ & 72 & $(64,9)$ \\
\hline Plástico & 1 & $(0,7)$ & 0 & $(0,0)$ & 0 & $(0,0)$ \\
\hline
\end{tabular}


$\mathrm{Na}$ localidade de Monte Alegre foram examinados $94,87 \%$ da população cadastrada (111/117 indivíduos). Entre os 111 indivíduos examinados, 98,2\% (109/11) estavam parasitados por ao menos uma espécie enteroparasita, comensal ou não, $70,3 \%$ (78/111) estavam parasitados por geohelmintos, 92,8\% (103/111) estavam parasitados por protozoários e $74,8 \%$ (83/111) estavam poliparasitados (infectados por ao menos três espécies enteroparasitas) (Tabela 4).

Ao analisar a prevalência para cada espécie enteroparasita pesquisada, foi constatado que $29,7 \%$ (33/111) dos indivíduos examinados estavam parasitados por Ascaris lumbricoides, 18,9\% (21/111) por Trichuris trichiura, 55,9\% (62/111) por ancilostomídeos, 64,0\% (71/111) por Entamoeba histolytica, 51,4\% (57/111) por Entamoeba coli, 21,6\% (24/111) por Giardia lamblia e 88,3\% (98/111) por Endolimax nana. Não foram encontrados indivíduos parasitados por Schistosoma mansoni, Strongyloides stercoralis, Enterobius vermicularis e Taenia sp. (Tabela 5).

$\mathrm{Na}$ localidade da Batateira foram examinados $91,53 \%$ da população cadastrada (54/59 indivíduos). Entre os 54 indivíduos examinados, 96,3\% (52/54) albergavam ao menos uma espécie enteroparasita comensal ou não, $81,5 \%$ (44/54) estavam parasitados por geohelmintos, 94,4\% (51/54) por protozoários e 79,6\% (43/54) estavam poliparasitados (Tabela 4).

Ao analisar a prevalência para cada espécie enteroparasita pesquisada, foi constatado que 46,3\% (25/54) dos indivíduos examinados estavam parasitados por Ascaris lumbricoides, 68,5\% (37/54) por Trichuris trichiura, 35,2\% (19/54) por ancilostomídeos, 66,7\% (36/54) por Entamoeba histolytica, 46,3\% (25/54) por Entamoeba coli, 22,2\% (12/54) por Giardia lamblia e 87,0\% (47/54) por Endolimax nana. Não foram encontrados indivíduos parasitados por Schistosoma mansoni, Strongyloides stercoralis, Enterobius vermicularis e Taenia sp. (Tabela 5).

$\mathrm{Na}$ localidade de Garapuá foram examinados $94,2 \%$ da população cadastrada (146/155 indivíduos). Entre as 146 crianças (0-14 anos) examinadas, $74,7 \%$ (109/146) estavam parasitadas por ao menos uma espécie enteroparasita, comensal ou não, 34,2\% (50/146) estavam parasitadas por geohelmintos, 70,5\% (103/146) estavam parasitadas por protozoários e $31,5 \%$ (46/146) estavam poliparasitadas (Tabela 4).

Ao analisar a prevalência para cada espécie enteroparasita pesquisada, foi constatado que 9,6\% (14/146) dos indivíduos examinados estavam parasitados por Ascaris lumbricoides, 28,1\% (41/146) por Trichuris trichiura, 11\% (16/146) por ancilostomídeos, 21,2\% (31/146) por Entamoeba histolytica, $19,9 \%$ (29/146) por Entamoeba coli, 29,5\% (43/146) por Giardia lamblia e 54,1\% (79/146) por Endolimax nana. De forma comum às demais populações estudadas, não foram encontrados indivíduos parasitados por Schistosoma mansoni, Strongyloides stercoralis, Enterobius vermicularis e Taenia sp. (Tabela 5).

Tabela 4. Resultados Laboratoriais (Valores Globais).

\begin{tabular}{|c|c|c|c|c|c|c|}
\hline \multirow[t]{2}{*}{ Variáveis } & \multicolumn{2}{|c|}{$\begin{array}{l}\text { Garapuá } \\
N=146\end{array}$} & \multicolumn{2}{|c|}{$\begin{array}{c}\text { Batateira } \\
\mathrm{N}=54\end{array}$} & \multicolumn{2}{|c|}{$\begin{array}{c}\text { Monte Alegre } \\
\quad \mathrm{N}=111\end{array}$} \\
\hline & $\mathbf{n}$ & $\%$ & $\mathbf{n}$ & $\%$ & $\mathbf{n}$ & $\%$ \\
\hline Parasitados & 109 & $(74,7)$ & 52 & $(96,3)$ & 109 & $(98,2)$ \\
\hline Prevalência de Helmintos & 50 & $(34,2)$ & 44 & $(81,5)$ & 78 & $(70,3)$ \\
\hline Prevalência de Protozoários & 103 & $(70,5)$ & 51 & $(94,4)$ & 103 & $(92,8)$ \\
\hline Poliparasitados & 46 & $(31,5)$ & 43 & $(79,6)$ & 83 & $(74,8)$ \\
\hline
\end{tabular}


Tabela 5. Resultados Laboratoriais (Prevalências das Espécies Diagnosticadas).

\begin{tabular}{l|c|c|c|c|c|c}
\multirow{2}{*}{ Variáveis } & \multicolumn{2}{c|}{$\begin{array}{c}\text { Garapuá } \\
\text { N= 146 }\end{array}$} & \multicolumn{2}{c|}{$\begin{array}{c}\text { Batateira } \\
\text { N= 54 }\end{array}$} & \multicolumn{2}{c}{$\begin{array}{c}\text { Monte Alegre } \\
\text { N = 111 }\end{array}$} \\
\cline { 2 - 7 } & $\mathbf{N}$ & $\mathbf{\%}$ & $\mathbf{n}$ & $\mathbf{\%}$ & $\mathbf{n}$ & $\mathbf{\%}$ \\
\hline Ascaris lumbricoides & 14 & $(9,6)$ & 25 & $(46,3)$ & 33 & $(29,7)$ \\
Trichuris trichiura & 41 & $(28,1)$ & 37 & $(68,5)$ & 21 & $(18,9)$ \\
Ancilostomídeos & 16 & $(11,0)$ & 19 & $(35,2)$ & 62 & $(55,9)$ \\
Entamoeba histolytica & 31 & $(21,2)$ & 36 & $(66,7)$ & 71 & $(64,0)$ \\
Entamoeba coli & 29 & $(19,9)$ & 25 & $(46,3)$ & 57 & $(51,4)$ \\
Giardia lamblia & 43 & $(29,5)$ & 12 & $(22,2)$ & 24 & $(21,6)$ \\
Endolimax nana & 79 & $(54,1)$ & 47 & $(87,0)$ & 98 & $(88,3)$ \\
\hline
\end{tabular}

\section{DISCUSSÃO}

Os resultados laboratoriais encontrados nas três localidades estudadas comprovam que a soma dos fatores ambientais (clima quente e úmido e solo poroso), socioculturais e demográficos (baixa escolaridade, utilização de fontes superficiais, defecação a céu aberto e aglomeração de indivíduos nas residenciais), econômicos (baixa renda, com reflexos na infraestrutura sanitária e tipologia construtiva do domicílio) e sanitários (carências ou inexistência de serviços públicos de saneamento básico) é favorável à transmissão de parasitas intestinais.

De forma geral, as prevalências encontradas nesta pesquisa, sobretudo nas comunidades quilombolas, são extremamente altas, quando comparadas às prevalências encontradas em estudos similares realizados em áreas urbanas pauperizadas e/ou em áreas rurais (MORAES, 1996; ASAOLU et al., 2002; CARNEIRO et al., 2002; PRADO et al., 2003; CHECKLEY et al., 2004; TEIXEIRA; HELLER; BARRETO, 2007; CABRAL-MIRANDA; DATTOLI; DIAS-LIMA, 2010; FONSECA et al., 2010).

As prevalências de indivíduos parasitados por geohelmintos nas localidades de Batateira e Monte Alegre foram mais elevadas que os percentuais médios estimados em municípios com baixo Índice de Desenvolvimento Humano (BRASIL, 2012).

Em todas as localidades estudadas, Endolimax nana foi a espécie de enteroparasita mais frequentemente encontrada. Ressalta-se que, apesar da baixa importância clínica, as altas prevalências de Endolimax nana encontradas nas diferentes localidades estudadas devem ser entendidas como um importante indicador epidemiológico da insalubridade ambiental, uma vez que sinaliza a existência de fatores favoráveis à transmissão de parasitas intestinais com via de transmissão fecal-oral, sujeito a uma menor influência de "vícios" relacionados ao uso de vermífugos.

São alarmantes os percentuais de indivíduos poliparasitados, sobretudo nas comunidades quilombolas da Batateira e Monte Alegre, em razão do prejuízo individual, no que se refere às possíveis manifestações clínicas resultantes, e dos prejuízos coletivos relacionados ao atraso nos níveis educacionais com repercussões no desenvolvimento econômico, e no sentido mais amplo, no alcance da cidadania plena.

A baixa discrepância dos valores globais encontrados nas localidades de Monte Alegre e Batateira corrobora a limitação do suprimento de água de baixa qualidade e do atendimento parcial da comunidade por vaso sanitário no controle das parasitoses intestinais (MORAES; CANCIO; CAIRNCROSS, 2004; HELLER, 2006). Vale ressaltar que além do atendimento parcial da comunidade de Monte Alegre por vaso sanitário, muitas vezes este equipamento sanitário é subutilizado, devido às práticas culturais enraizadas.

As prevalências encontradas podem estar subestimadas, devido à utilização de apenas um método de análise laboratorial. Além do método laboratorial, a análise de apenas uma amostra por indivíduo pode ter levado a resultados em falsonegativos, devido a questões fisiológicas, como a excreção descontínua de ovos e cistos. 
Nas diferentes localidades estudadas foi observada uma maior prevalência da infecção por protozoários. Fatores como o consumo de água de fontes superficiais sem qualquer tratamento prévio, a alta aglomeração de pessoas nos domicílios e os programas de tratamento em massa com antihelmínticos podem explicar os resultados encontrados.

Os resultados encontrados reforçam que a política nacional de investimento público em saneamento básico em municípios de pequeno porte, com especial atenção às comunidades tradicionais, é um instrumento fundamental a minorar os impactos sociais e ambientais resultantes dos ideais neoliberais que permeiam o Estado capitalista.

Vale ressaltar que, devido a população de apenas 15.374 habitantes e a existência de populações quilombolas e extrativistas, o município de Cairu é parte dos municípios alvo do Programa de Aceleração do Crescimento 2 (PAC 2 Saneamento), que prevê até 2014, investimentos da ordem de 4 bilhões de reais em ações de saneamento básico em municípios com população até $50 \mathrm{mil}$ habitantes, com prioridade ao atendimento de comunidades indígenas, quilombolas e extrativistas.

No escopo das ações de cunho curativo, os resultados laboratoriais encontrados nesta pesquisa classifica o município de Cairu como alvo do Plano Integrado de Ações Estratégicas de Eliminação da Hanseníase, Filariose, Esquistossomose, Como Problema de Saúde Pública, Tracoma Como Causa de Cegueira e Controle das Geohelmintíases que possui como meta a redução da pobreza no País, por meio do controle ou eliminação, até 2015, deste conjunto de doenças (BRASIL, 2012).

\section{CONCLUSÃO}

Espera-se que os resultados encontrados nesta pesquisa possibilitem uma maior visibilidade à situação de injustiça socioambiental diagnosticada, determinada pela ausência do Estado na provisão de políticas sociais básicas.
Em paralelo, espera-se que os índices sofríveis do nível de cobertura pelos serviços públicos de saneamento básico e os impactos diagnosticados na saúde pública sejam utilizados pela sociedade civil como um instrumento de luta por políticas que priorizem investimentos e a gestão pública dos serviços com controle social.

As prevalências encontradas denotam a urgência no planejamento e implementação de intervenções médico-curativas aliadas a programas de educação em saúde e melhorias na infraestrutura sanitária, no ambiente público e no ambiente domiciliar.

Ressalta-se que a universalização dos serviços públicos de saneamento básico nas três localidades estudadas depende da participação direta do Poder Publico Municipal, somado a um alto protagonismo da sociedade civil. A sustentabilidade econômica das intervenções passa pelo emprego de tecnologias individuais, principalmente, no que se refere à destinação final das águas residuais.

Os resultados gerados nesta pesquisa devem ser utilizados como subsídio ao planejamento, implementação e avaliação de políticas sociais necessárias à garantia da proteção e promoção à saúde, bem-estar e qualidade de vida nas localidades estudadas.

É necessário o desenvolvimento de novas pesquisas para aprofundar a compreensão dos fatores de risco associados às parasitoses intestinais em populações tradicionais assentadas no território municipal, possibilitando uma maior eficácia das medidas de controle.

\section{AGRADECIMENTOS}

Agradecemos a Prefeitura Municipal de Cairu-Bahia pelo apoio técnico e financeiro, necessários ao desenvolvimento da pesquisa que gerou os dados para esse artigo.

\section{REFERÊNCIAS BIBLIOGRÁFICAS}

ASAOLU, S.O.; OFOEZIE, I. E.; ODUMUYIWA, P. A.; SOWEMIMO, O. A.; OGUNNIYI. T. A. B. Effect of water supply and sanitation on the prevalence and intensity of Ascaris lumbricoides among pre-school-age children in Ajebandele and Ifewara, Osun State, Nigeria. Transactions of the Royal Society of Tropical Medicine and Higyene. v. 96, p. 600-604, 2002. 
BRASIL. Plano integrado de ações estratégicas de eliminação da hanseníase, filariose, esquistossomose e oncocercose como problema de saúde pública, tracoma como causa de cegueira e controle das geohelmintíases : plano de ação 2011-2015. Ministério da Saúde, Secretaria de Vigilância em Saúde, Departamento de Vigilância em Doenças Transmissíveis. Brasília, 2012.

CABRAL-MIRANDA,G.; DATTOLI, V. C. C; DIAS-LIMA, A. Enteroparasitos e Condições Socioeconômicas e Sanitárias em uma Comunidade Quilombola do Semiárido Baiano. Revista de Patologia Tropical. v. 39, n. 1, p. 48-55, jan./mar. 2010.

CARNEIRO, F. F.; CIFUENTES, E.; TELLEZ-TOJO, M. M.; ROMIEU, I. The risk of Ascaris lumbricoides infection in children as an environmental health indicator to guide preventive activities in Caparaó and Alto Caparaó, Brazil. Bulletin of the World Health Organization. v. 80, n.1, p. 4046, 2002.

CHECKLEY, W.; GILMAN, R. H.; BLACK, R. E.; EPSTEIN, L. D.; CABRERA, L., STERLING, C. R.; MOULTON, L. H. Effect of water and sanitation on childhood health in a poor Peruvian peri-urban community. The Lancet, v. 1363, p.112-118, jan. 2004.

FONSECA, E. O. L.; TEIXEIRA, M. G.; BARRETO, M. L.; CARMO, E. H.; COSTA, M. C. N. Prevalência e fatores associados às geo-helmintíases em crianças residentes em municípios com baixo IDH no Norte e Nordeste brasileiros. Cad. Saúde Pública. v. 26, n. 1, p.143-152, jan. 2010, Rio de Janeiro.

HELLER, L. Acesso aos serviços de abastecimento de água e esgotamento sanitário no Brasil: considerações históricas, conjunturais e prospectivas. Centro de Estudos Brasileiros. Universidade de Oxford. Paper n. 73, jun. 2006.

MENEZES, L. C. C. (1984) Considerações sobre saneamento básico, saúde pública e qualidade de vida. Revista de Engenharia Sanitária, v. 23, n. 1, p. 55-61, jan./mar.

MORAES, L. R. S.; CANCIO, J. A.; CAIRNCROSS, S. Impact of drainage and sewerage on intestinal nematode infections in poor urban areas in Salvador, Brazil. The Royal Society of Tropical Medicine and Hygiene, v. 98, p. 197-204, 2004.

MORAES, L. R. S.; REIS, M. D. G. C.; ZANTA, V. M.; LUZ, L. D. D.; ORRICO, S. R. M.; MACHADO, N. L.; AGRA-FILHO, S. S.; NASCIMENTO, S. A. D. M.; SILVA, B. J. D. (2006) Plano municipal de saneamento ambiental de Alagoinhas, Brasil: Metodologia e Resultados. In: SIMPÓSIO LUSO-BRASILEIRO DE ENGENHARIA SANITÁRIA E AMBIENTAL, 12., 2006, Figueira da Foz. Anais... Lisboa: APRH; APESB; ABES. 14p.

Organização Panamericana da Saúde (2011). Agua y Saneamiento. Evidencias para políticas públicas con enfoque em derechos humanos y resultados em salud pública. Washington, D. C.
PRADO, M. S.; STRINA, A.; BARRETO, M. L.; OLIVEIRA-ASSIS, A. M.; PAZ, L. M.; CAIRNCROSS, S. Risk factors for infection with Giardia duodenalis in pre school children in the city of Salvador, Brazil. Epidemiol. Infect, v. 131, p. 899-906, 2003

TEIXEIRA, J. C.; HELLER, L.; BARRETO, M. L. Giardia duodenalis infection: risk factors for children living in substandard settlements in Brazil. Cad. Saúde Pública. Rio de Janeiro, v. 23, n. 6, p.1489-1493, jun. 2007.

TEIXEIRA, J.B. (2011) Saneamento rural no Brasil. In: Panorama do Saneamento Básico no Brasil, v.7. HELLER, L.; MORAES, L.R.S.; BRITTO, A.L.; BORJA, P.C.; REZENDE, S.C.R. Brasília: Ministério das Cidades/Secretaria Nacional de Saneamento Ambiental. p. 220-279.

WHO; UNICEF (2012). Joint Monitoring Programme for Water Supply and Sanitation. Washington, D.C. 